\title{
Integración de las tecnologías de información y comunicación en la docencia universitaria para una educación inclusiva
}

\section{Integration of information and communication technologies in university teaching for inclusive education}

\author{
Alejandra Mercedes Colina Vargas \\ Universidad ECOTEC, Ecuador \\ Autor para correspondencia: acolina@ecotec.edu.ec \\ Fecha de recepción: 24 de septiembre de 2018 - Fecha de aceptación: 15 octubre de 2018
}

Resumen: El artículo que se presenta tiene como objetivo analizar cómo la integración de las TIC en el currículo universitario favorece la generación de aprendizaje en contextos sociales diversos y procesos cognitivos emergentes. Los referentes teórico que sustentarón la investigación se tomaron de fuentes documentales que explicitan las características del objeto de estudio desde diferentes posiciones teóricas, lo que permitió asumir una postura crítica que alecciona a la integración y posterior apropiación de las TIC en la docencia universitaria con una visión social e inclusiva, donde emerge el constructor de que la apropiación se concibe como la integración plena de las TIC en el contexto cultural y social de los docentes. Del contexto presentado, se afirma que el docente tiene la capacidad de usar las TIC al punto de volverlas importantes para sus actividades cotidianas en el aula y así generar competencias en TIC que contribuyen de manera significativa al mejoramiento de sus condiciones de vida y su transformación social (Colina, 2018). Ante este argumento, se afirma que el desarrollo de la inclusión educativa reclama de un análisis pertinente de las prácticas educativas con sus correspondientes procesos de transformación, no reduciéndose como un discurso puntual con recorrido temporal limitado, puesto que han sido muchas las intenciones declaradas y los reglamentos escritos que se han puesto en marcha en múltiples contextos para esta modalidad educativa. Finalmente, se alcanzó una sistematización y reflexiones en las definiciones de integración y apropiación de las tecnologías para favorecer la inclusión educativa, especificándose en el docente universitario por ser un elemento de vital importancia con miras a contribuyendo a la mejora y preparación de estudiantes autónomos, críticos del conocimiento y de la sociedad adquiriendo una formación profesional.

Palabras Claves: integración; tecnologías de información y comunicación; educación superior; educación inclusiva

Abstract: The article presented aims to analyze how the integration of ICT in the university curriculum favors the generation of learning in diverse social contexts and emerging cognitive processes. The theoretical referents that supported the research were taken from documentary sources that explain the characteristics of the object of study from different theoretical positions, which allowed us to assume a critical stance that teaches the integration and subsequent appropriation of ICT in university teaching with a vision social and inclusive, where the builder emerges that the appropriation is conceived as the full integration of ICT in the cultural and social context of teachers. From the context presented, it is stated that the teacher has the ability to use ICT to the point of making them important for their daily activities in the classroom and thus 
generate ICT skills that contribute significantly to the improvement of their living conditions and their social transformation (Hill, 2018). Given this context, it is affirmed that the development of educational inclusion requires a relevant analysis of educational practices with their corresponding processes of transformation, not being reduced as a punctual discourse with a limited time course, since there have been many declared intentions and written regulations that have been implemented in multiple contexts for this educational modality. Finally, a systematization and reflections were reached in the definitions of integration and appropriation of technologies to favor educational inclusion, specifying in the university teacher for being an element of vital importance with a view to contributing to the improvement and preparation of autonomous, critical students of knowledge and society by acquiring professional training. Key Words: integration; information and communication technologies; higher education; inclusive education

\section{Introducción}

La integración de las Tecnologías de Información y Comunicación (TIC) en la educación superior emerge en la actualidad como un desafío en la dinámica tradicional del profesor universitario pues trae consigo cambios en el papel que desempeñaba dejando de ser la única fuente de información con su exposición magistral, y se convierte en un mediador entre el alumno y los contenidos de aprendizaje.

Es por ello, que las instituciones de educación superior constituyen hoy en día una institución social, la cual ha estado influenciada por los cambios producidos a partir de los avances científicos y tecnológicos pasando por el desarrollo del conocimiento y la información, por dar un ejemplo en el plano social, siendo loable resaltar el apoyo que han dado a la socialización y formación de las nuevas generaciones; ella se encarga de transmitir los conocimientos que una sociedad considera indispensables para su desarrollo y las tradiciones y cultura que identifica al grupo social al que sirve. Durante casi todo el siglo XX han sido vistas como espacios aislado de la realidad social en la que la visión reduccionista, atomista y antropocéntrica del mundo se refleja en la organización del currículum.

En este contexto de profundos cambios y transformaciones socio-económicas, tecnológicas y culturales, exige de la educación universitaria que esté en condiciones de desarrollar las competencias requeridas para la comprensión de estos cambios, que estimulen la creatividad, así como una clara concepción sobre el futuro frente a los retos del actual milenio. Se requiere una formación universitaria que no esté centrada en la transmisión de la información sino en la formación de capacidades y actitudes básicas orientadas hacia la creatividad, la resolución de problemas y la formación en valores. (Falco, 2017)

En este marco de idea, se concibe un nuevo modelo de crecimiento basado en la tecnología y el conocimiento, en la cual las instituciones se organizan para generar, adoptar, adaptar, difundir y usar los conocimientos, ya sean científicos, tecnológicos y gerenciales. De hecho, se pretende un desarrollo social y económico en función de los conocimientos de las personas a fin de crear valor e innovaciones que se traduzcan en productos y servicios, en definitiva, bienestar para el ciudadano. 
De la misma manera, se enfrentan a nuevos desafíos de expandir y renovar permanentemente el conocimiento, de dar acceso universal a la información y promover la capacidad de comunicación entre individuos y grupos sociales, entre otros. Para ellos se han planteado políticas educacionales en algunos países de la Latinoamérica, que tomen en cuenta la incorporación de las Tecnologías de Información y Comunicación (TIC) en los establecimientos educativos tanto en los procesos de enseñanza- aprendizaje como en la organización de la tarea docente, constituyéndose, no como una simple moda o una mera sofisticación, sino una respuesta a las necesidades de desarrollo de nuestros países y de inserción en el mundo globalizado.

Partiendo de esa base Sánchez (2008) destaca que, las TIC no son suficientes ni ineludibles para alcanzar el desarrollo humano, se requiere que éstas sean encausadas a fin de que asuman un papel social al servicio del desarrollo de los pueblos y, ante todo, de los sectores más demandadas. Es por ello, que se debe superar el desafío de discernir cuándo y en qué condiciones estas tecnologías pueden aportar al desarrollo humano, pues su sola presencia no soluciona el problema, sino que es necesario ir más allá de la conectividad, promoviendo el acceso equitativo, uso y apropiación social de los recursos disponibles.

Desde este contexto, los nuevos propósitos que desempeñan las TIC en la transformación de las universidades las obligan a reorientar y redefinir los procesos de integración de estas herramientas en los planes y programas de estudio de pregrado y postgrados. De igual manera, la profesión docente está cambiando desde un enfoque centrado en el profesor y basado en clases magistrales, hacia una formación centrada principalmente en el estudiante dentro de un entorno interactivo de aprendizaje, donde produzca, socialice y apropie de forma critica el conocimiento. Esto debido a que el conocimiento es abierto, inconcluso, y está en constante reconstrucción. Antes este escenario, se plantea para el docente universitario el reto de utilizar, integrar y apropiar la tecnología en su práctica docente como un medio a favor de un desarrollo humano y social más inclusivo que considera los diferentes aspectos del desarrollo como elementos centrales de la transición hacia sociedades de la información. (Claro, 2011)

De igual manera, otro de los retos que debe enfrentar las instituciones educativas en la actualidad lo constituye el hecho de que éstas deben brindar una respuesta educativa pertinente a las características y necesidades educativas particulares de cada estudiante, y estar en capacidad de educar a todos los estudiantes en el respeto a sus diferencias, se conoce como Educación Inclusiva. (Viquez, 2014)

Se consideran las nuevas tecnologías como un medio excepcional de acceder a personas con limitaciones físicas y psicológicas, se han desarrollado grandes esfuerzos en el uso de herramientas para ayudar a personas con problemas visuales, auditivos, de aprendizaje y otras limitaciones a integrarse y permanecer en el sistema educativo. El problema está vinculado a la posibilidad de que queden excluidas de la cultura digital; es decir, que impedimentos visuales o auditivos, limitaciones de aprendizaje o de otro tipo no les permitan interactuar con un aparato tecnológico. Por otra parte, se presenta una oportunidad que tiene relación con la potencialidad de estas tecnologías para adaptarse y constituirse en tecnologías de apoyo que respondan a las necesidades especiales de los aprendices (Claro, 2011). 
Por lo antes expuesto el presente artículo pretende analizar cómo la integración de las TIC en el currículo universitario favorece la generación de aprendizaje en contextos sociales diversos y procesos cognitivos emergentes que garanticen la inclusión de todos los estudiantes.

El artículo presenta inicialmente referentes teórico en el cual se sustentó la investigación, posteriormente presenta las conclusiones.

\section{Métodos}

Esta investigación se ubica dentro de un estudio cualitativo de corte descriptivo y exploratorio. Dado el carácter de estudio exploratorio, se inició con la revisión sistemática de literatura a partir de la compilación de resúmenes, artículos científicos y estudios previos, los cuales fueron analizados minuciosamente destacando las áreas que tributan más a la investigación.

Se llevó a cabo la búsqueda y definición de palabras clave en bases de datos y revistas científicas de alto impacto de temas individuales para finalmente sintetizar y analizar lo encontrado. Para la descripción e indagación se efectuó un análisis de material bibliográfico recabado en diferentes fuentes especializadas. Se aplicaron métodos teóricos inductivo deductivo para el análisis de la información mostrando las reflexiones y análisis crítico de la integración de las TIC en la docencia universitaria favorece una educación inclusiva.

\section{Referentes teóricos}

\section{Apropiación de las TIC y la educación superior}

Específicamente para el caso de estudio que ocupa este artículo, se tomó en consideración de acuerdo a lo formulado por Cardón (2006), la connotación de la apropiación en el ámbito de la tecnología; señalando que el mismo se remonta a las preocupaciones de los investigadores que formaron el núcleo constitutivo de los primeros estudios de uso de las tecnologías de información y comunicación (TIC), definiéndolo como:

Aquella actividad social, cultural, económica y política de pleno derecho. El uso de las tecnologías, en efecto, se inscribe profundamente en la vida social de las personas y es reductor considerar el impacto de las tecnologías de la información como un simple asunto de costo, de funcionalidad o de simplicidad de las interfaces. (sp.)

De igual manera, el autor destaca que la apropiación referida a las tecnologías fue promovida inicialmente por los franceses y francocanadiense en los años 70 y 80, para ese entonces se pretendía desarrollar una postura sociopolítica de los usos sobre la dimensión conflictiva de dicho término en el seno de las relaciones de producción y de reproducción de la economía capitalista. En este sentido, la noción de apropiación a lo cual hace alusión permite describir el proceso de interiorización progresiva de competencias técnicas y cognitivas obrantes entre los individuos y los grupos que manejan cotidianamente estas tecnologías. Colina (2018)

\section{La Educación inclusiva y las TIC}


La UNESCO establece en sus documentos que la educación inclusiva se conoce como el proceso de identificar y responder a la diversidad de las necesidades de todos los estudiantes a través de la mayor participación en el aprendizaje, la cultura y las comunidades, y reduciendo la exclusión en la educación, se aspira la búsqueda de la equidad e igualdad de derechos entre todas las personas. La inclusión es definida por Echeíta (2009: 381) como "un proceso de mejora e innovación interminable pues conlleva un constante cambio social que supone continuos esfuerzos siempre susceptibles de mejora" a fin de responder a las necesidades particulares de cada estudiante a fin garantizar un ambiente educativo inclusivo.

La inclusión es una mirada a la aceptación de la diversidad, puesto que las diferencias son inherentes al ser humano y están presentes en cualquier proceso de enseñanza - aprendizaje, permitiendo conocer y defender los derechos que le corresponden a cada persona. Incluir signfica aceptar a todos tal cual como son, sin tener en cuenta razas, culturas, condicion física, social, etc (Watts y Lee, 2017).

La educación inclusiva según Laitón, Gómez, Sarmiento y Mejía (2017) pretende garantizar la participación de todos los estudiantes en el proceso de enseñanza- aprendizaje cotidiano haciendo uso de las TIC se evita la exclusión en las instituciones educativas debido a dificultades sociales, actitudinal, económica o por los métodos de enseñanza empleados por los docentes.

La educación inclusiva constituye un proyecto que propone construir una educación que rechace cualquier tipo de exclusión y que potencie la participación y el aprendizaje equitativo, por tanto, su implementación en el contexto escolar, es una tarea titánica dado las exigencias propias de la sociedad actual que requiere de una educación universal, igualitaria y de calidad (Watts y Lee, 2017).

En el contexto de la relación entre la educación inclusiva y las TIC se tiene por un lado que los docentes se encuentran ante un reto en la sociedad actual de responder a las necesidades particulares de cada estudiante siendo capaces de reconocerlos como aprendices diferentes para personalizar a cada uno de los estudiantes a fin de garantizar un ambiente educativo inclusivo que propicie prácticas educativas inclusivas que permitan solventar las barreras de aprendizaje propias de cada estudiante anticipándose con estrategias y proyectos educativos innovadores.

Por otro parte, el uso de las TIC en el aula pretende beneficiar a los estudiantes ofreciéndoles un ambiente escolar que tome en cuenta la diversidad con "una educación más equitativa, igualitaria y adaptada a su realidad; gozando de la autonomía e iniciativa en la adquisición de conocimiento y desarrollo de competencias" (Laitón, Gómez, Sarmiento y Mejía, 2017).

Es así como Moya (2009) señala que la incorporación de las TIC en las aulas permite nuevas formas de acceder, generar y transmitir información y conocimientos, así como también una flexibilización de tiempo y espacio para el desarrollo de la practica educativa para transformar la enseñanza y mejorar la participación y el aprendizaje de todos.

\section{El nuevo rol del docente y las TIC}


Al hacer referencia a la inclusión de las TIC en la educación universitaria, se hace necesario destacar en este contexto que los docentes pasan a ser proveedores de recursos, diseñadores de su propio material, organizadores de los espacios de aprendizaje, tutororientador-facilitador e investigador, con el fin de lograr la formación de ciudadanos responsables en todos los aspectos de la vida humana.

Existe una realidad a nivel global los docentes a menudo no son capaces de dar respuesta a las necesidades educativas especiales, pues ello trae consigo cambios en la metodología de trabajo y en el currículo. Aunado a esto, no disponen de recursos necesarios para atender la diversidad, lo cual merma la calidad de la educación. Sin embargo, los estudiantes con necesidades especiales tienen derecho como los otros a asistir a las instituciones de educación ordinarias (Watt y Lee, 2017).

Por tanto, con la integración de las TIC como un todo en el currículo universitario se permitiría la generación de aprendizaje en contextos sociales diversos y procesos cognitivos emergentes, los cuales sería imposible a través del uso de estrategias convencionales. En tal sentido, en la búsqueda de religar, acepción esta acuñada por Morin (1998) a los fines de significar unir o ligar, en este caso, la teoría a favor del aprendizaje se requiere la conjunción de los fenómenos en todas sus complejidades sin deducirlo a un pensamiento lineal, sino que vincular en forma concreta la presencia de las TIC hacia la mejora de la educación universitaria.

Este profesional de la docencia está dedicado a cumplir sus actividades al servicio de la sociedad a través de las instituciones educativas universitarias donde laboran. Es así, que Bendito, Ferrer y Ferreres (1995) resaltan que los profesores universitarios deben ser reflexivos, críticos, competentes en el ámbito de su disciplina, y estar capacitado para ejercer la docencia y desempeñar labores de investigación. De igual manera, "deben intentar desarrollar una actividad docente, comprometida con la idea de potenciar el aprendizaje de los estudiantes" contribuyendo a la mejora y preparación de estudiantes autónomos, críticos del conocimiento y de la sociedad adquiriendo una formación profesional.

Del contexto antes descrito, se requieren políticas que favorezcan la inclusión de todos los estudiantes sin distinción y formación para que los docentes y la comunidad educativa en general, sepan tratar apropiadamente las diferentes necesidades presentes en el aula, todo en pro de la inclusión y atención a la diversidad (Watts y Lee, 2017).

Así lo confirman Suárez, Almerich, Gargallo y Aliaga (2010) al decir:

Dentro del proceso de integración de las TIC en el aula, el profesorado se configura como uno de los elementos clave, sin el cual dicho proceso presentaría más dificultades, o no tendría lugar. Por ello, ha de estar capacitado para poder utilizar las TIC, adquiriendo los conocimientos y habilidades necesarios que le permitan la utilización de éstas como un recurso en su proceso de enseñanza-aprendizaje (p.3).

Las TIC constituyen herramientas que promueven factores de equidad, pertinencia e inclusión educativa en los ambientes de aprendizaje y favorecen el desarrollo de habilidades como razonar, inferir, interpretar, argumentar, proponer, modelar, tomar decisiones, resolver 
problemas, entre otros. De allí que su uso como mediador de aprendizaje incluso en personas en situación de discapacidad, es primordial para alcanzar la calidad educativa en cualquier nivel (Watts y Lee, 2017).

En palabras de Cabero y Fernández (sf.) las TIC ayudan a una serie de aspectos como son: poner en acción mejores o nuevos aprendizajes, establecer con ellas innovaciones pedagógicas y cambios organizacionales, facilitar los procesos de comunicación y la ruptura de la unidad de tiempo, espacio y acción, que es donde, por lo general, se desarrolla la acción formativa tradicional. Asi como también, destacan que en los últimos años las percepciones que se tiene en relación a la aplicación de las TIC en los procesos formativos han ido variando, dándose actualmente tres diferentes formas de aplicarlos, no contrapuestas sino complementarias: TIC (Tecnologías de la Información y la Comunicación), TAC (Tecnologías para el Aprendizaje y el Conocimiento) y TEP (Tecnologías para el Empoderamiento y la Participación).

Donde la perspectiva TIC, comprende el uso de recursos fundamentalmente divisados como facilitadores y transmisores de información, así como recursos educativos dirigidos a los estudiantes, los cuales pueden ser adaptados a las necesidades y características independientes de los sujetos, pudiendo conseguir con ellos una verdadera formación audiovisual, multimedia e hipertextual. A los cuales el docente debe conocer los aspectos tecnológicos e instrumentales de dichos recursos.

Por su parte, la visión TAC, se concibe como instrumentos facilitadores del aprendizaje y la difusión del conocimiento. Son por tanto vistas, no tanto como instrumentos de comunicación, sino como herramientas para la realización de actividades para el aprendizaje y el análisis de la realidad circundante por el estudiante. Su uso se orienta hacia usos más formativos, tanto para docentes como para estudiantes, con el objetivo de aprender de manera más significa y excelente.

Mientras que, el enfoque TEP, se percibe no como meros recursos educativos, sino como instrumentos para la participación y la colaboración de docentes y estudiantes que, además, no tienen que estar situados en el mismo espacio y tiempo. Se parte por tanto de la perspectiva de que el aprendizaje no sólo tiene una dimensión individual, sino también social, ya que la formación implica aprender en comunidad y ser capaz de interactuar y colaborar para construir el conocimiento.

Adell en García y López (2011) establece cinco funciones primordiales de las TIC como herramienta de apoyo y eje principal para la innovación educativa inclusiva, como son:

1.) Acceso: Aprender a utilizar correctamente la tecnología.

2.) Adopción: Apoyar a una forma tradicional de enseñar y aprender.

3.) Adaptación: Integración en formas tradicionales de clase.

4.) Apropiación: Uso colaborativo, proyectos y situaciones necesarias. 
5.) Innovación: Descubre nuevos usos de la tecnología y combinan las diferentes modalidades.

Las TIC favorecen una atención personalizada (Cabero, 2008), expone las TIC ayudan a superar las limitaciones que se derivan de las discapacidades cognitivas, sensoriales y motóricas del alumnado, en concreto:

- Ayudan a superar las limitaciones que se derivan de las discapacidades cognitivas, sensoriales y motóricas del alumnado.

- Favorecen la autonomía de los estudiantes, pudiéndose adaptar a las necesidades y demandas de cada alumno o alumna de forma personalizada.

- Ofrecen un feed-back inmediato.

- Favorecen la comunicación sincrónica y asincrónica de estos estudiantes con el resto de compañeros y el profesorado.

- Ahorran tiempo para la adquisición de habilidades y capacidades en los estudiantes.

- Favorecen el diagnóstico de los alumnos y alumnas.

- Respaldan un modelo de comunicación y de formación multisensorial.

- Propician una formación individualizada, y el que los alumnos puedan avanzar a su propio ritmo, lo cual es de extremada importancia para los sujetos con algún tipo de discapacidad.

- Favorece el desarrollo de la autonomía e independencia de las personas.

- Evitan la marginación, la brecha digital, que introduce el verse desprovisto de utilizar las herramientas de desarrollo de la sociedad del conocimiento.

- Facilitan la inserción sociolaboral de aquel alumnado con dificultades específicas.

- Proporcionan momentos de ocio.

- Ahorran tiempo para la adquisición de habilidades y destrezas.

- Propician el acercamiento de estas personas al mundo científico y cultural, y el estar al día en los conocimientos que constantemente se están produciendo.

- Y favorece la disminución del sentido de fracaso académico y personal.

Cabero (2008) el incorporar en el aula de clase las TIC trae consigo una serie de ventajas y desventajas de carácter general que aportan los multimedia y la telemática para apoyar la enseñanza los cuales se presentan en el Cuadro 1. 
Ventajas y desventajas de incorporar en el aula las TIC como apoyo a la educación inclusiva.

\begin{tabular}{|c|c|}
\hline Ventajas & Desventajas \\
\hline Alto poder motivante & $\begin{array}{l}\text { Uso depende de la discapacidad (visual, auditiva, motórica, } \\
\text { cognitiva, entre otros) }\end{array}$ \\
\hline $\begin{array}{l}\text { Facilita la creación de entornos } \\
\text { dinámicos y atractivos }\end{array}$ & Integración depende del tipo de discapacidad y del grado \\
\hline $\begin{array}{l}\text { Flexibilización en los entornos de } \\
\text { comunicación }\end{array}$ & $\begin{array}{l}\text { Uso dependerá estará sujeto desde el punto de vista del hardware y } \\
\text { del software }\end{array}$ \\
\hline $\begin{array}{l}\text { Repetición de la información y los } \\
\text { ejercicios } \\
\text { Adaptabilidad a las características } \\
\text { individuales de los estudiantes }\end{array}$ & $\begin{array}{l}\text { Para la integración se debe realizar un análisis multidisciplinario } \\
\text { (pedagogos, ingenieros, psicólogos, diseñadores, etc.) }\end{array}$ \\
\hline
\end{tabular}

\section{Cuadro 1.}

Fuente: elaboración propia a partir de Cabero (2008) 


\section{Discusión}

Los autores revisados coinciden en que los procesos relacionados con la educación inclusiva deben llevar consigo la participación y presencia de todos los estudiantes en igualdad de oportunidades en el proceso de aprendizaje independiente de cual sea su condición (Mas y Olmos, 2012; Cabero, 2008; Claro, 2011; Echeíta, 2009).

La actitud de los docentes, siendo ellos los actores claves en el proceso educativo, en torno la inclusión va marcada de factores relacionados con los problemas educativos de los estudiantes tomando en consideración la discapacidad, su naturaleza y grado; asi como también por la falta de preparación o capacitación en relación al diagnóstico y abordaje de estudiantes con necesidades educativas especiales en las aulas de clase.

Las TIC permiten aumentar las posibilidades de estos alumnos para relacionarse con el entorno, y mejorar, de esta forma, su calidad de vida afectiva, personal, emocional, laboral y profesional, evitando la exclusión y favoreciendo, por tanto, la igualdad en el contexto de la educación superior no debe estar ajena a los procesos de cambios y transformaciones que requiere la sociedad hoy, involucrando al docente universitario en su mejora y desarrollo profesional que atienda la diversidad e inclusión educativa como una competencia clave en su función docente.

Resalta que aún persisten en el contexto educativo universitario las actitudes negativas hacia la discapacidad percibida como una de las principales barreras de la integración.

Enfatiza las competencias del perfil docente del profesor universitario para atender a una educación inclusiva donde destacan: la capacidad crítica y reflexiva, la gestión de las situaciones de aprendizaje, la promoción del aprendizaje cooperativo, la comunicación, la mejora continuada, la detección de necesidades individuales y grupales, la tutoría, la orientación y el asesoramiento (Mas y Olmos, 2012). 


\section{Conclusión}

La apropiación de las TIC en la docencia universitaria trae consigo desafíos que deben ser superados a partir de un cambio metodológico de la forma tradicional de enseñanza y la creación de nuevas estrategias en pro de garantizar una educación de calidad e inclusiva. Se requiere para ello el desarrollo por parte de los docentes de unas prácticas educativas soportadas en nuevas propuestas que apunten hacia la innovación con el uso e incorporación de las TIC y la inclusión de todos en el sistema educativo.

Para ello los docentes deben tener dominio de las TIC en los procesos pedagógicos que permite la innovación educativa en espacios inclusivos, pues si desconoce los beneficios que ofrecen las herramientas tecnológicas aun teniendo los recursos en el aula de clase, difícilmente va a lograr adaptar estos espacios educativos a favor de los estudiantes, y minimizar las barreras de aprendizaje y propiciar el éxito del aprendizaje en todos los estudiantes va a ser un trabajo agotador para el docente, pues el entorno de aprendizaje resultara tedioso.

En la actualidad, es de conocimiento general que el desarrollo de la inclusión educativa requiere de un análisis constante de las prácticas educativas y de los procesos de actualización educativa, no pudiendo reducirse simplemente a una política o estrategia institucional puntual con carácter temporal limitado, puesto que han sido muchas las intenciones declaradas y los reglamentos escritos que se han puesto en marcha en múltiples contextos para esta modalidad educativa y aun son pocos los impactos o beneficios tangibles de ellos. Indica que la educación inclusiva pretende modificar "la sociedad" haciéndola más justa, más tolerante, y más humana.

Se plantea como necesidad demandante de la sociedad un docente universitario que posea un perfil amplio incluya el diagnostico, diseño, desarrollo, comunicación, tutorización, evaluación, innovación, participación activa en todo lo relacionado con la atención a la diversidad e inclusión de estudiantes con necesidades educativas especiales.

Están las instituciones de educación superior o universidades preparadas como elemento de reflexión final con políticas institucionales que faciliten la formación y actualización del docente para la atención a estos estudiantes con necesidades educativas especiales como un elemento prioritario. 


\section{Bibliografía}

Benedito, V, Ferrer, V. y Ferreres, V. (1995). La Formación universitaria a debate. Universidad de Barcelona, España. [Libro en línea] Disponible: http://books.google.co.ve/books?id=QV141BClMiwC\&printsec=frontcover \&dq=la+form acion+universitaria+al+debate \&hl=es\&sa=X\&ei=U0MoUc2LO4qy9gSpioDwBg\&ved $=0$ CDAQ6AEwAA

Cabero, J. (2008) TICs para la igualdad: la brecha digital en la discapacidad. Revista ANALES Universidad Metropolitana 15 Vol. 8, No 2 (Nueva Serie). Recuperado en: https://www.researchgate.net/publication/233926363_TICs_para_la_igualdad_la_brecha_ digital_en_la_discapacidad

Cabero, y Fernandez (sf.) Una mirada sobre las TIC y la Educación Inclusiva. Reflexión en torno al papel de las TIC en la Educación Inclusiva. Recuperado de: http://www.centrocp.com/una-mirada-sobre-las-tic-y-la-educacion-inclusiva/

Cardón, D. (2006). La innovación por el uso. En: Ambrosi, A., Peugeot, V. y Pimienta, D. Palabras en juego: enfoques multiculturales sobre las sociedades de la información. C\&F Editions. Recuperado de: http://www.casanas.com.ar/artsAdj/Palabras_en_juego221.pdf

Claro, M. (2011). El papel de las tecnologías de la información y las comunicaciones en la educación inclusiva. Santiago de Chile: CEPAL. Documento en línea. Disponible: https://repositorio.cepal.org/handle/11362/3937

Colina, A. (2018). Apropiación De Las TIC. Docencia Universitaria Y Pensamiento Complejo. Universidad Nacional Experimental "Rafael María Baralt". Tesis Doctoral.

Echeita, G. (2009). “Los dilemas de las diferencias en la educación escolar”. En Verdugo, M.A. et altri. "Mejorando resultados personales para una vida de calidad. VII Jornadas Científicas de Investigación sobre Personas con Discapacidad”. Salamanca: Amarú. pp. 381-395.

García, M., López, R. (2012). Explorando, desde una perspectiva inclusiva, el uso de las TIC para atender a la diversidad. Profesorado Revista de curriculum y formación del profesorado, 16(1), 277-293.

Hernández, P. (2003). Formación Docente en Educación Superior: la experiencia de un modelo de intervención. Artículo en línea. Disponible: http://www.contextoeducativo.com.ar/2003/3/nota-05.htm

Johnson, C. (1993). Aprendizaje Colaborativo, referencia virtual del Instituto Tecnológico de Monterrey, México Disponible: http://campus.gda.itesm.mx/cite

Laitón Z,, E., Gómez A., S., Sarmiento P., R., y Mejía C., C. (2017). Competencia de prácticas inclusivas: las TIC y la educación inclusiva en el desarrollo profesional docente. Sophia, 13 (2), 82-95 
Llorente C., M. (2005). Tutoría virtual .Documento en línea. Disponible: http://tecnologiaedu.us.es/cuestionario/bibliovir/tutoriavirtual.pdf

Martínez, M. (2004). Ciencia y Arte en la metodología cualitativa. México: Trillas.

Mass T., O., Olmos R., P. (2012). La atención a la diversidad en la educación superior: Una perspectiva desde las competencias docentes. Revista Educación Inclusiva Vol. 5, $\mathrm{N}^{\circ} 1$. Recuperado en: http://www.ujaen.es/revista/rei/linked/documentos/documentos/15-12.pdf

Morín, E. (1998). Introducción al pensamiento complejo. Editorial Gedisa. España

Moya, A. (2009). Las nuevas tecnologías en la educación. Revista Digital de Innovación y Experiencias Educativas, (45), 1-9.

Organización de las Naciones Unidas para la Educación, la Ciencia y la Cultura (UNESCO, 2008), en el informe "Los docentes y la enseñanza en un mundo en mutación".

Sánchez P., José (2008). La Docencia Universitario. Purga para un manifiesto antipedagógico. Universidad Salesiana de Ecuador. [Libro en línea] Disponible: http://books.google.co.ve/books?id=UkhqJWjKNYYC\&pg=PA2\&lpg=PA2\&dq=la+doc encia+universitaria+de+un+manifiesto\&source=bl\&ots=SxfE4EF0y\&sig=ZuB3u3EjOa5VExGFsQD6wxq763s\&hl=es\&sa=X\&ei=Z0IoUbRI5Cy9gS7_IHwCw\&ved=0CDIQ6AEwAQ

Suárez, J.M. Almerich, G. Gargallo, B. y Aliaga, F (2010) "Las competencias en TIC del profesorado y su relación con el uso de los recursos tecnológicos" Archivos Analíticos de Políticas Educativas, 18 (10). Disponible: http://epaa.asu.edu/ojs/article/view/755

Torres, A. (2004). "Necesidad de reforma universitaria y educación abierta y a distancia" Documento en línea. Disponible: http://www.unidad094.upn.mx/revista/39/reforma.htm

Víquez B., I. (2014). La Educación Inclusiva es una propuesta para que las instituciones educativas tanto públicas como privadas. Congreso Iberoamericano de Ciencia, Tecnología, Innovación y Educación. Universidad de Costa Rica. Disponible: https://www.oei.es/historico/congreso2014/memoriactei/675.pdf

Watts, C. y Lee, L (2017). Las TIC como herramientas de inclusión educativa. Acta Scientiae Informaticae 1 (1), 91-97. 